\title{
GRÁFICO COMBINADO SHEWHART-CUSUM APLICADO A UM PROCESSO DE ROTULAGEM DE GARRAFAS
}

\section{COMBINED SHEWHART-CUSUM CHART APPLIED TO A PROCESS OF LABELING BOTTLES}

\author{
Elisa Henning ${ }^{1}$; Robert Wayne Samohyl ${ }^{2}$; Olga Maria Formigoni Carvalho Walter ${ }^{3}$; Rodrigo Leite \\ Lindoso 4 \\ ${ }^{1}$ Universidade do Estado De Santa Catarina - UDESC - Santa Catarina - Brasil \\ dma2eh@joinville.udesc.br \\ ${ }^{2}$ Universidade Federal de Santa Catarina - UFSC - Santa Catarina - Brasil \\ samohyl@yahoo.com \\ ${ }^{3}$ Universidade Federal de Santa Catarina - UFSC - Santa Catarina - Brasil \\ olgaformigoni@gmail.com \\ ${ }^{4}$ Universidade do Estado De Santa Catarina - UDESC - Santa Catarina - Brasil \\ rodrigoleite91@gmail.com
}

\begin{abstract}
Resumo
Os tradicionais gráficos do tipo Shewhart são recomendados na detecção de grandes alterações na média de um processo e, os gráficos de soma cumulativa (CUSUM) na sinalização de pequenas e moderadas alterações. Todavia, se a magnitude da mudança for desconhecida ou se modifica ao longo do tempo, nenhum dos gráficos mencionados irá ter um bom desempenho para todas as situações. Uma solução possível é combinar múltiplos gráficos para abranger mudanças de diversas magnitudes. Assim, um gráfico combinado Shewhart-CUSUM corresponde à inclusão de novos parâmetros probabilísticos a um gráfico de somas acumuladas (CUSUM). Esta combinação tem como objetivo aumentar a sensibilidade do gráfico CUSUM para alterações de maior magnitude, buscando um modelo mais eficiente de gráfico de controle. Este artigo traz uma aplicação de um gráfico combinado Shewhart-CUSUM binomial em um processo de rotulagem de garrafas, conduzido por meio de um estudo de caso. Foi comparado seu desempenho com os gráficos CUSUM e Shewhart individuais. O gráfico combinado se mostrou eficiente, pois sinalizou tanto alterações repentinas de maior magnitude, quanto um aumento menor, mas gradativo $e$ persistente no número de não conformidades, não sinalizados pelos gráficos individuais.
\end{abstract}

Palavras-Chave: Gráfico combinado Shewhart-CUSUM, distribuição binomial, rotulagem de garrafas.

\section{Introdução}

A busca da qualidade e a melhoria desta é um fator presente nas atividades industriais e de serviços. A qualidade, de um produto ou serviço, é um dos mais importantes fatores de decisão dos consumidores na seleção de empresas e organizações que competem entre si. Mesmo onde não há concorrência direta, como por exemplo, em serviços públicos, é inegável a importância da 
qualidade na realização destes. Assim uma organização, pública ou privada, que deseja gerar produtos e serviços com qualidade deve investir em atividades permanentes de monitoramento e controle.

As atividades de monitoramento e controle são fundamentais neste sentido. A eliminação sistemática de desperdícios diversos, que acarreta em menores custos, maior produtividade e consequentemente um lucro maior, é uma das ações que são esperadas quando se trabalha na melhoria contínua da qualidade. $\mathrm{O}$ aumento da satisfação do consumidor e uma melhor reputação da organização são consequências comuns quando os objetivos, traçados com base no material coletado pelo monitoramento e controle, são alcançados.

Estas atividades de monitoramento e controle necessitam de técnicas formais apropriadas para que obtenham êxito e, sob esta ótica, os métodos estatísticos desempenham papel importante. Com a aplicação de técnicas estatísticas investiga-se a variabilidade de um processo e a partir destas ações executa-se o controle como forma de redução de falhas e aumento da confiabilidade. Esta atividade é muito mais efetiva do que apenas procurar eliminar os defeitos (ROTONDARO, 2002). Para controlar a variabilidade, para Mari (1997) métodos estatísticos transformam dados do processo em estatísticas, assim o problema é resolvido estatisticamente e então os resultados são transformados em dados do processo. É um ciclo que busca melhorar continuamente.

Os métodos estatísticos e sua aplicação na melhoria da qualidade têm uma longa história. $\mathrm{Na}$ década de 20, Walter Shewhart, desenvolveu o conceito estatístico de gráficos de controle, dando um início formal ao campo do controle estatístico de qualidade. Os gráficos eram simples, voltados à atividade de inspeção, direcionado ao "chão de fábrica" (MONTGOMERY, 2004).

Os gráficos de controle utilizados no monitoramento de características de qualidade de interesse representadas por variáveis contínuas, originárias de um processo de medição, são chamados de gráficos de controle por variáveis. Citam-se aqui gráficos para a média, a amplitude e a variância de um processo. Em muitas situações, no entanto, as características de qualidade de interesse não podem ser mensuradas por grandezas físicas. Nestes casos, a qualidade pode ser representada pela proporção média de produtos defeituosos produzidos ou pela frequência de ocorrência de defeitos. Os gráficos de controle usados para monitorar estas medidas de qualidade são denominados gráficos de controle por atributos.

Os gráficos do tipo Shewhart são considerados excelentes para a detecção de grandes desvios na média do processo, ou de aumentos significativos na variância ou da fração defeituosa, e alcançaram grande sucesso devido à facilidade da regra de decisão. Esta se baseia apenas no exame do último ponto analisado, ou seja, se ele está além dos limites de controle do gráfico, neste caso deve-se intervir no processo. Esta é também uma grande desvantagem, pois ignora qualquer informação passada dada pela sequência anterior de pontos, tornando gráfico do tipo Shewhart 
relativamente insensível a pequenas mudanças no processo, da ordem de 1,5 $\sigma$ (erros padrão) ou menos (WOODALL, 1986; ALVES; SAMOHYL, 2004; COSTA; EPPRECHT; CARPINETTI, 2004).

As regras suplementares (ou sensibilizantes) aplicadas aos gráficos do tipo Shewhart procuram suplantar esta dificuldade, todavia reduzem a simplicidade e facilidade de uso (ROCHA, 2004). Estas regras foram criadas com o propósito de acelerar a detecção de alterações no processo. Muitas delas tentam formalizar a percepção de que algo, no processo, possa estar fora de ordem. A incorporação dessas regras implica no aumento do risco $\alpha$, ou seja, há um aumento no número de alarmes falsos (COSTA; EPPRECHT; CARPINETTI, 2004).

Assim, o gráfico de controle das Somas Acumuladas (CUSUM) é uma alternativa para este problema, sendo recomendados ao monitoramento de processos sujeitos a pequenas alterações. Neste gráfico a decisão sobre o estado do controle estatístico do processo é baseada na informação acumulada das diversas amostras anteriores, e não somente na última delas, sendo possível sinalizar com maior rapidez os pequenos desajustes, assim como identificar no tempo o momento em que ocorre uma mudança no processo (ALVES; SAMOHYL, 2004).

Assim, os gráficos do tipo Shewhart são recomendados na detecção de grandes alterações na média e variabilidade de um processo e gráficos CUSUM na sinalização de pequenas a moderadas alterações. Todavia se a magnitude da mudança for desconhecida ou se modifica ao longo do tempo, nenhum dos gráficos mencionados irá ter um bom desempenho para todas as situações. Uma solução possível é combinar múltiplos gráficos para abranger mudanças de diversas magnitudes, como por exemplo, adicionar limites de Shewhart a um gráfico CUSUM (TSUNG; WANG, 2010).

Este artigo traz uma aplicação de um gráfico combinado Shewhart-CUSUM binomial a um processo de rotulagem de garrafas e tem como objetivo, a partir de um estudo retrospectivo, analisar o desempenho de um gráfico combinado comparando-o com os gráficos individuais. A partir daqui o artigo está assim estruturado: na seção 2 descrevem-se brevemente os gráficos combinados Shewhart-CUSUM, na seção 3 estão os procedimentos metodológicos adotados, na seção 4 os resultados da aplicação realizada. Finalizando, na seção 5 estão as conclusões e considerações finais.

\section{Gráficos combinados Shewhart-Cusum}

$\mathrm{Na}$ seção anterior foi dito que a combinação de múltiplos gráficos permite detectar mudanças de magnitudes diversas. Deste modo, uma abordagem para aumentar a sensibilidade do gráfico CUSUM, para grandes mudanças, é o procedimento combinado Shewhart-CUSUM (MONTGOMERY, 2004). Este procedimento é uma modificação simples do CUSUM, com a adição dos limites de controle do gráfico do tipo Shewhart. Um gráfico combinado Shewhart- 
CUSUM incorpora num mesmo eixo os valores observados, os limites de controle Shewhart, a estatística CUSUM e o limite de controle do CUSUM.

Este gráfico pressupõe, de um modo geral, quatro linhas de controle: os limites superiores e inferiores de Shewhart ( $L S C$ e $L I C)$; o limite de controle $h\left(h^{+}\right.$e $\left.h^{-}\right)$; os valores das estatísticas correspondentes às amostras (observadas ou padronizadas) e os valores das somas cumulativas $C_{+}$ (positiva) e $C$-(negativa). Algumas reduções podem ser feitas de acordo com o caso a ser tratado. Por exemplo, se apenas é desejada a investigação do problema, um gráfico unilateral superior pode ser suficiente (ROCHA, 2004). A Figura 1 ilustra um gráfico combinado Shewhart-CUSUM unilateral superior.

Figura 1 - Exemplo de gráfico combinado unilateral superior.

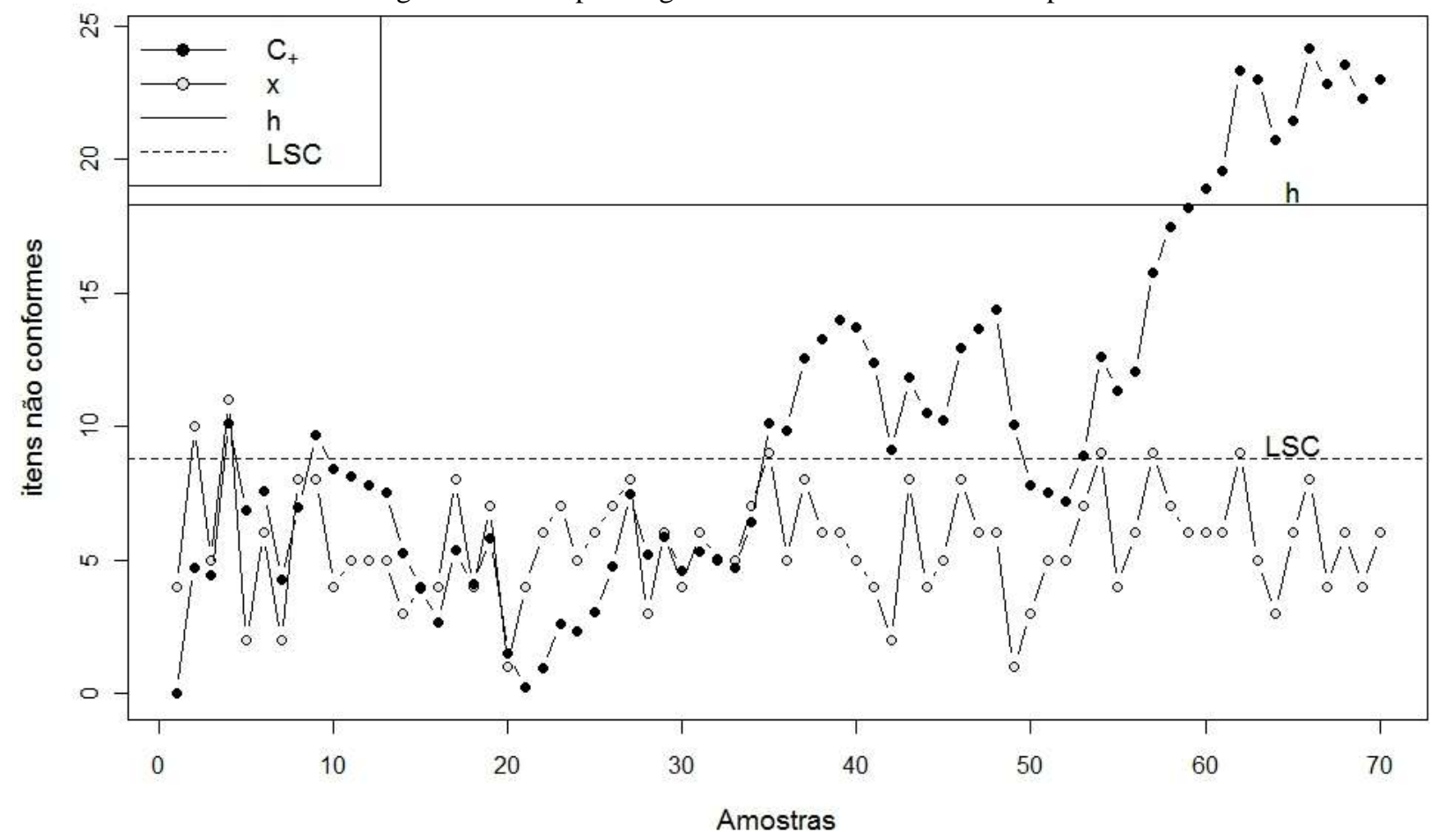

Fonte: Adaptado de Morais (2002)

Antes de prosseguir, é necessário definir alguns parâmetros que medem o desempenho de um gráfico de controle. Este desempenho em geral é mensurado em termos do número de amostra antes da sinalização de uma alteração. O ARL (Average Run Length) corresponde ao valor esperado de amostras coletadas até emissão de um sinal, verdadeiro ou não. Num gráfico de controle o $A R L_{0}$ indica o número médio de amostras coletadas até a emissão de um sinal durante o período sob controle, ou seja, está relacionado à emissão de um alarme falso (erro tipo I). Já o $A R L_{l}$ representa o número médio de amostras coletadas até a emissão de um sinal que indique uma situação de fora de controle, quando esta realmente ocorreu, ou seja, um alarme verdadeiro (ALVES; SAMOHYL, 2004).

A primeira citação de um gráfico de controle combinado foi feita no trabalho de Westgard et al (1977), que aplicou o gráfico combinado como uma alternativa ao CUSUM, para tentar melhorar 
o controle em laboratórios de análises clínicas. Lucas (1982) efetuou uma análise comparativa do comportamento desses três tipos de gráficos de controle, Shewhart, CUSUM e Shewhart-CUSUM, para média de processo com distribuição normal. Para o mesmo valor de $h$ o combinado sinaliza antes que um gráfico CUSUM alterações maiores, mas a custa de mais alarmes falsos. Assim, Lucas (1982) sugeriu um pequeno aumento no valor de $h$. Este aumento em $h$ significa um valor um pouco maior do $A R L_{0}$ de projeto, ou seja, uma diminuição na taxa de alarmes falsos. Com esta modificação, o gráfico combinado sinaliza antes que o CUSUM mudanças maiores que $2 \sigma$ aproximadamente.

Outras aplicações do gráfico combinado para médias abrangem o monitoramento de processos laboratoriais (SOLBERG, 1984; BLACKSELL et al, 1994; 1996; ROCHA, 2004; CONRATHS; SCHARES, 2006), monitoramento ambiental (USEPA, 1989; GIBBONS, 1999; CHOU; O'BRIEN; BARNETT, 2001; ZHOU et al, 2008) e monitoramento dos erros de previsão (SOUZA; SAMOHYL, 2008).

\subsection{Gráfico combinado Shewhart-Cusum binomial}

A literatura apresenta poucos trabalhos sobre gráficos combinado para atributos. Resultados numéricos relativos ao gráfico combinado superior unilateral CUSUM-Shewhart, considerando dados com distribuição de Poisson podem ser encontrados em Yashchin (1985), Abel (1990), Hawkins e Olwell (1998), Morais (2002) e Morais e Pacheco (2006).

Um gráfico combinado Shewhart-CUSUM binomial unilateral superior corresponde a um gráfico CUSUM binomial com adição do limite superior $(L S C)$ de um gráfico $n p$ do tipo Shewhart. Assim, seja uma série de amostras $Y_{i} \operatorname{com} i \in Z^{+}$, independentes, de tamanho $n$, com distribuição binomial, o gráfico combinado Shewhart-CUSUM binomial é obtido plotando-se as estatísticas $C_{i}$ e $X_{i}$, em relação à amostra $Y_{i}$,

$$
C_{i}=\max \left(0, C_{i-1}+X_{i}-k\right)
$$

onde $C_{i}$ é a estatística CUSUM com $C_{0}=u, 0 \leq u \leq h ; X_{i}$ é o número de itens não conformes na amostra $Y_{i}$ com $i=1,2, \ldots ; k$ é uma constante denominada de valor de referência do CUSUM e depende da magnitude da mudança que se deseja detector e $h$ é limite superior de controle do CUSUM. Se o processo está sob controle, as somas acumuladas definidas em (1) descrevem um percurso aleatório com média zero. Porém, se a média muda para algum valor acima, então uma tendência ascendente se revelará na soma acumulada $C_{i}$.

Já, o limite superior $(L S C)$ do gráfico $n p$ do tipo Shewhart convencional é sumarizado por

$$
L S C=n p+3 \sqrt{n p(1-p)}
$$


onde $p$ corresponde a proporção $p_{0}$. Estes limites, conhecidos como $3 \sigma$ (ou convencionais), são obtidos através de uma aproximação à distribuição normal.

São então traçadas duas linhas, $C_{i}$ e $X_{i}$, e dois limites de controle, $h$ e $L S C$ (Limite Superior de Shewhart). Se $C_{i}$ ultrapassa o limite superior de controle $h$, ou $X_{i}$ ultrapassa o limite superior $L S C$, o que ocorrer primeiro, diz-se que o processo está fora de controle estatístico.

O parâmetro $k$, de gráficos CUSUM para contagens, neste trabalho, é determinado pela taxa de contagem aceitável e a taxa de contagem que se deseja detectar, sendo sumarizado pela Equação (3) (GAN, 1993).

$$
k=\frac{n \ln \left(\left(1-p_{0}\right) /\left(1-p_{1}\right)\right)}{\ln \left(\left(1-p_{0}\right) /\left(1-p_{1}\right)-\ln \left(p_{0} / p_{1}\right)\right)},
$$

onde $n$ é o tamanho da amostra, $p_{0}$ a proporção sob controle estatístico e $p_{1}$ a proporção considerada fora de controle estatístico, ou seja, aquela que espera que o gráfico sinalize rapidamente.

Os limites de controle $h$ e $L S C$ serão determinados a partir da probabilidade de ocorrência de alarmes falsos (erro tipo I) do gráfico combinado. A combinação de gráficos de controle, considerando a função de probabilidade de ocorrência de alarmes falsos, foi abordada por Montgomery (2004), Souza e Samohyl (2008), Coelho (2008) e Wu et al (2008). De modo geral, se há $r$ variáveis estatisticamente independentes para um produto em particular, e se o gráfico com probabilidade de erro tipo I (alarmes falsos) é mantido para cada uma, então a verdadeira probabilidade (erro tipo I) para cada procedimento de controle conjunto, é $\alpha=1-(1-\alpha)^{r}$ onde $(1-\alpha)^{r}$ é a probabilidade de que todas as variáveis sejam representadas no gráfico simultaneamente dentro de seus limites de controle (MONTGOMERY, 2004; ALVES, 2009). Seguindo este raciocínio, quando dois gráficos diferentes monitoram a mesma variável, a taxa de alarmes falsos do gráfico combinado $\alpha_{c s}$ será uma combinação de taxas individuais de cada um, $\alpha_{s}$ e $\alpha_{c}$ para Shewhart e CUSUM respectivamente. Assim, uma expressão que sumariza essa combinação é dada por :

$$
\alpha_{c s}=\alpha_{s}+\alpha_{c}-\alpha_{s} \alpha_{c}
$$

A partir da determinação das taxas de alarme falso do gráfico combinado e das partes individuais, podem-se calcular os limites da parte CUSUM e do gráfico tipo Shewhart separadamente, aplicando o método que for mais conveniente ao pesquisador. No planejamento de um gráfico combinado deve-se então ajustar o $L S C$ (do gráfico tipo Shewhart) e o limite superior $h$ do CUSUM, de modo a alocar o erro tipo I (taxa de alarmes falsos). 
Neste trabalho, o limite superior h do CUSUM será determinado a partir dos trabalhos de Reynolds e Stoumbos (1999, 2001), aproximados pela função LambertW (HENNING, 2010; HENNING; SAMOHYL; ALVES, 2010) de acordo com a Equação (5)

$$
\begin{aligned}
h \approx & \frac{1}{r_{2}}\left(-\left(\frac{n r_{2} \gamma}{e^{-n r_{2} \gamma}-1}\right)+n A R L_{0}\left(p_{0} r_{2}-r_{1}\right)-\left[L_{1}-L_{2}+\frac{L_{2}}{L_{1}}+\frac{L_{2}\left(L_{2}-2\right)}{2 \mathrm{~L}_{1}^{2}}+\frac{L_{2}\left(6-9 \mathrm{~L}_{2}+2 \mathrm{~L}_{2}^{2}\right)}{6 \mathrm{~L}_{1}^{3}}\right]\right), \\
& \frac{\left(1-2 \mathrm{p}_{0}\right)}{3}
\end{aligned}
$$

com

$L_{1}=-\ln \left(-\left(\frac{1}{e^{n r_{2} \gamma}-1}\right)\left(e^{\left.n r_{2} \gamma+n\left(r_{1}-p_{0} r_{2}\right)\left(-A R L_{0}-\frac{r_{2} \gamma}{\left(1-e^{-n r_{2} \gamma}\right)\left(r_{1}-p_{0} r_{2}\right)}\right) n r_{2} \gamma\right)}\right)\right.$

$L_{2}=\ln \left(-L_{1}\right)$

$\operatorname{com} r_{1}=-\ln \left(\frac{1-p_{1}}{1-p_{0}}\right) ; r_{2}=-\ln \left(\frac{p_{1}\left(1-p_{0}\right)}{p_{0}\left(1-p_{1}\right)}\right)$ e $\gamma=\frac{r_{1}}{r_{2}}$

Os limites da parte Shewhart podem ser os convencionais (Equação 2) ou então probabilísticos (exatos), isto é, calculados a aplicando-se a distribuição binomial, e não a aproximação à normal.

A aplicação dos gráficos de controle de Shewhart e CUSUM para dados com distribuição binomial só será eficaz se os dados atenderem às seguintes premissas: os dados devem ter distribuição binomial, não apresentar superdispersão e devem ser independentes.

O distanciamento da distribuição binomial, a presença de superdispersão, que significa variância maior que $n p(1-p)$, além da existência de correlação entre as unidades amostrais, pode afetar o desempenho dos gráficos de controle, tanto de Shewhart, como o CUSUM, e consequentemente no gráfico combinado. Especificamente, com o processo sob controle, resulta em mais alarmes falsos.

A suposição mais importante relativa aos gráficos de controle é a independência das observações de um processo. Gráficos de controle convencionais não funcionam bem se a característica da qualidade apresenta níveis, ainda que pequenos, de correlação ao longo do tempo. Especificamente, com o processo sob controle, resulta em mais alarmes falsos (SHARMA, 2003; MONTGOMERY, 2004). Em relação às variáveis binomiais não se tem registros de artigos que 
tenham investigado com maior profundidade se a violação da aderência em um grau menor afeta o desempenho de um gráfico do tipo Shewhart $p$ ou $n p$. Para Jones e Govindaraju (2000) é possível estabelecer limites Shewhart sem verificar a aderência, mas recomendam a verificação para o caso de aparecerem muitos pontos além dos limites sem nenhuma explicação pelo processo. Wetherill e Brown (1991), todavia, recomendam verificar a aderência antes de estabelecer os limites de controle.

\section{Procedimentos metodológicos}

A aplicação do gráfico combinado foi feita com dados de um processo de rotulagem de garrafas de uma marca de bebida destilada, pertencente a uma empresa de grande porte.

É importante destacar que os trabalhos empíricos de aplicação de gráfico combinado Shewhart-CUSUM para atributos são relativamente escassos no país. Nesse sentido, o presente trabalho pretende trazer uma contribuição no aspecto empírico. A questão de pesquisa é associada a como se comporta o processo quando os dados são analisados por meio do gráfico combinado Shewhart-CUSUM. Nesse contexto, o presente trabalho utiliza como abordagem metodológica o estudo de caso, com base nos trabalhos de Voss, Tsikriktsis e Frohlich (2002) e Yin (2010).

A justificativa pela escolha da abordagem estudo de caso se dá em função de que o pesquisador não tem controle sobre os eventos (os dados não são simulados, ou seja, os dados monitorados são informações reais de um processo industrial, além de que a participação dos pesquisadores ocorreu como observadores passivos), o contexto é relevante e o tema é contemporâneo (pois nota-se uma discussão mais intensa na literatura ao longo dos últimos anos sobre competitividade e exigência do mercado consumidor com relação à qualidade dos produtos, além da necessidade de métodos de monitoramento e controle estatístico do processo que proporcionem melhor qualidade ao produto fabricado).

Os dados correspondem às unidades não conformes resultantes no processo de colocação dos rótulos e contra-rótulos nas garrafas, após o enchimento. O rótulo representa o conjunto de designações, ilustrações ou marcas que caracterizam a bebida. No rótulo devem estar informações obrigatórias como: marca, teor alcoólico, volume, aditivos, prazo de validade, composição e registros. O contra-rótulo é considerado uma extensão do rótulo (RIZZON; MENEGUZZO, 2006).

Quando o rótulo se apresentava rasgado, descentralizado, amassado, descolando ou com falha na impressão, considerava-se o produto defeituoso, devendo a garrafa ser lavada e retornada ao processo de rotulagem, o que era considerado retrabalho.

Na prática o desenvolvimento, implantação e utilização de gráficos de controle, passam por duas fases operacionais, a fase I e a fase II (SAMOHYL, 2009; VINING, 2009). A fase I é em geral um estudo retrospectivo, que consiste em obter-se uma amostra representativa dos dados com o 
objetivo de determinar os limites de controle. Nesta fase, serão verificadas as suposições necessárias, independência, ausência de superdispersão e aderência à distribuição binomial. Quando estas suposições são satisfeitas, os gráficos podem ser aplicados e conclusões tiradas sobre o estado de controle estatístico do processo. Esta fase, que corresponde à estimação de parâmetros do processo, só deve ser encerrada quando se tem a certeza de que o processo encontra-se estável e ajustado. A fase II tem o objetivo de monitorar o processo utilizando os limites de controle especificados na fase I (SAMOHYL, 2009).

Para a aplicação do gráfico foram utilizadas séries de dados de quatro dias consecutivos, sendo o primeiro para a fase I, e os três seguintes para a fase II. As amostras eram sequenciais, e uma caixa com 36 unidades era retirada de cada lote, totalizando aproximadamente 18 amostras por dia. Se houvesse indicação de estar fora de controle estatístico, o lote retornava, para avaliação e posterior lavagem e nova rotulação. O método de inspeção é visual. O conjunto de dados está no Anexo.

Para a verificação da autocorrelação foram aplicados os estimadores usuais das funções de autocorrelação e autocorrelação parcial amostrais (DU; LI, 1991; GAUTHIER; LATOUR, 1994; LATOUR, 1998; SILVA; SILVA, 2002; SILVA, 2005; CUI; LUND, 2009). Testes estatísticos formais foram aplicados para verificar a ausência de superdispersão e aderência à distribuição normal. Todo o tratamento estatístico dos dados foi feito com o GNU R (R DEVELOPMENT CORE TEAM, 2011), com o auxílio dos pacotes "qcc" (SCRUCCA, 2004), para o gráfico do tipo Shewhart com regras sensibilizantes e teste para verificar a superdispersão e "vcd" (MEYER; ZEILEIS; HORNIK, 2009) para os testes de aderência. As demais rotinas, para a construção dos gráficos CUSUM binomial e combinado, estão em Henning (2010).

\section{Resultados e análise}

Como as amostras eram sequenciais, no tempo, e pertenciam à mesma caixa, o processo de amostragem não dava nenhuma garantia de aleatoriedade. Todavia, os gráficos das funções de autocorrelação e autocorrelação parcial, Figura 2, indicam a ausência de autocorrelação no processo. Também se verificou a ausência de superdispersão ( $p$-valor=0,4044). A aderência à distribuição binomial foi avaliada mediante dois testes: qui quadrado ( $p$-valor=0,139) e baseado na razão de verossimilhança ( $p$-valor=0,116). 
Figura 2 - Gráficos da função de autocorrelação e autocorrelação parcial para os dados da rotulagem de garrafas
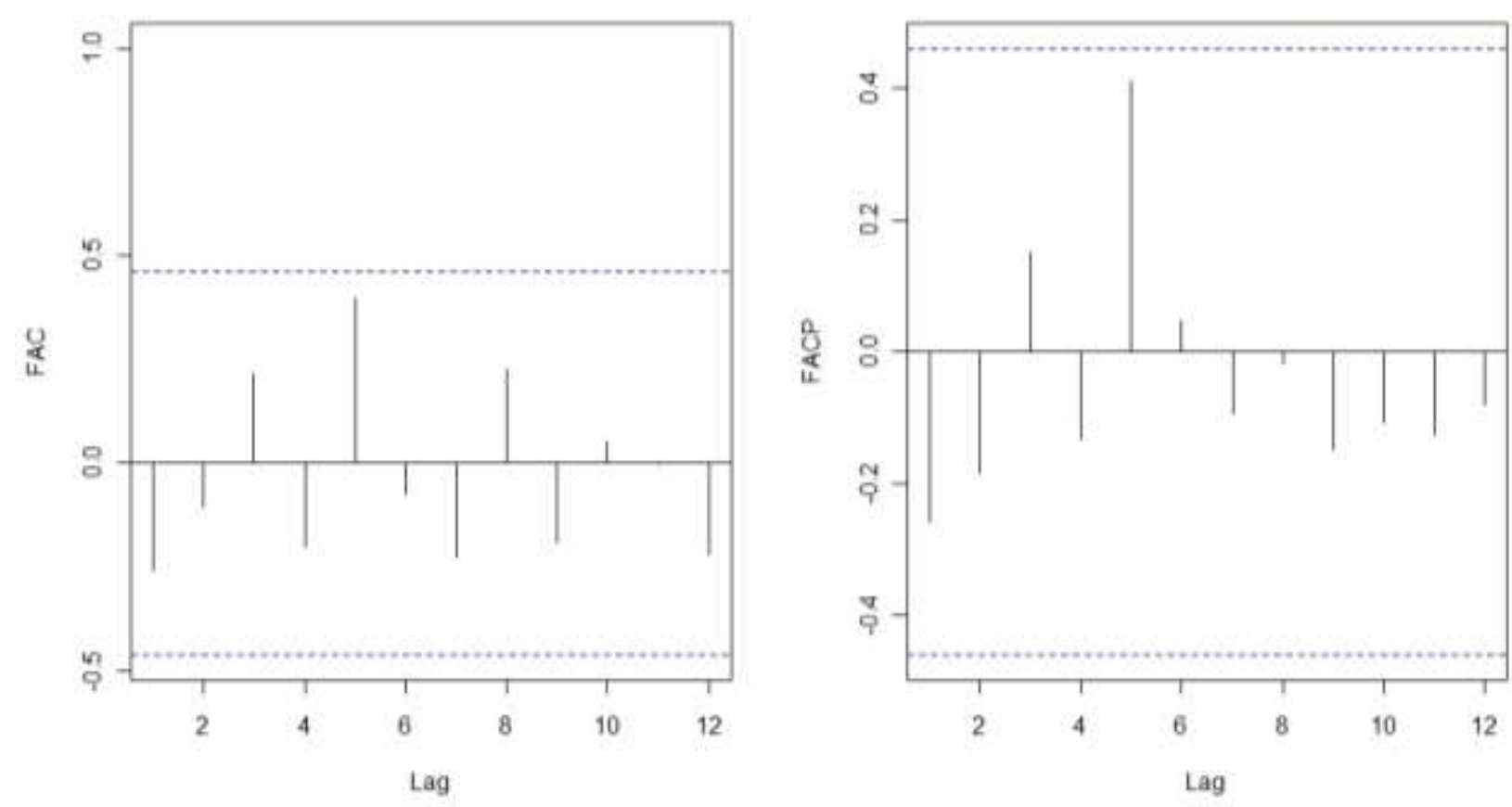

Fonte: Autoria própria (2011)

Conforme informação anterior a empresa aplicava gráficos de Shewhart com limites $3 \sigma$ convencionais $\left(A R L_{0}=370, \alpha=0,27\right)$ e $L S C=5,9$. O tamanho da amostra é $n=36$ e a proporção considerada sob controle, neste trabalho, é $p_{0}=0,052$. Este valor de $\operatorname{LSC}(5,9)$ não corresponde à probabilidade de ocorrência de alarmes falsos (Erro tipo I), pois $P(x \geq \operatorname{LSC}(5,9))=0,0023$, ou seja, $\alpha=0,23 \%$. Para fins de comparação, do combinado com os outros gráficos, inclusive o de Shewhart, o $A R L_{0}$ alvo adotado é de $443(\alpha=0,23)$. Assim o limite convencional $(L S C=5,9)$ é mantido e, está muito próximo do limite probabilístico $(L S C=6)$.

A probabilidade $p_{1}$, fora de controle, necessária para a parte CUSUM é $p_{1}=0,07$, um acréscimo de $37 \%$ em $p_{0}$. Para a definição deste valor considerou-se detectar uma mudança de cerca de $0,5 \sigma$ (meio desvio-padrão) na média $n p$. Optou-se também por um combinado com os limites de Shewhart não muito "largos". O gráfico é menos sensível a pequenas variações, mas acredita-se que vai captar mudanças moderadas a grandes. A intenção é não distanciar muito os limites da parte Shewhart do combinado do $L S C$ de um gráfico Shewhart individual. Assim a parte CUSUM tem $A R L_{0}=548, \operatorname{com} k=2,18$ e $h=11,3$. A parte Shewhart tem $A R L_{0}=2298, \operatorname{com} L S C=7$.

Na Figura 3 está o gráfico combinado Shewhart-CUSUM. A linha tracejada vertical (18 amostra), separa os períodos sob-controle (fase I) e a de monitoramento (fase II). A parte Shewhart do gráfico combinado emite um sinal na $27^{\mathrm{a}}$ amostra e depois na $41^{\mathrm{a}}$ amostra. Avaliando os dados, nestas duas situações, constata-se que $25 \%$ dos itens analisados são defeituosos. A parte CUSUM dá informações sobre a tendência do processo, indicando que há um aumento na média $n p$ de itens 
não conformes. A partir da $27^{\mathrm{a}}$ amostra o CUSUM não retorna ao zero, e verifica-se aos poucos uma tendência crescente. Nas amostras 41 e 45 a linha do CUSUM é ultrapassada, sendo que após esta última amostra o processo não retorna ao estado de controle. Há evidências que a média $n p$ do processo possa ter aumentado, o que resulta no deterioramento da qualidade do processo de rotulagem. Isto poderia implicar em retrabalho para a empresa.

Figura 3 - Gráfico Shewhart-CUSUM para os dados da rotulagem de garrafas

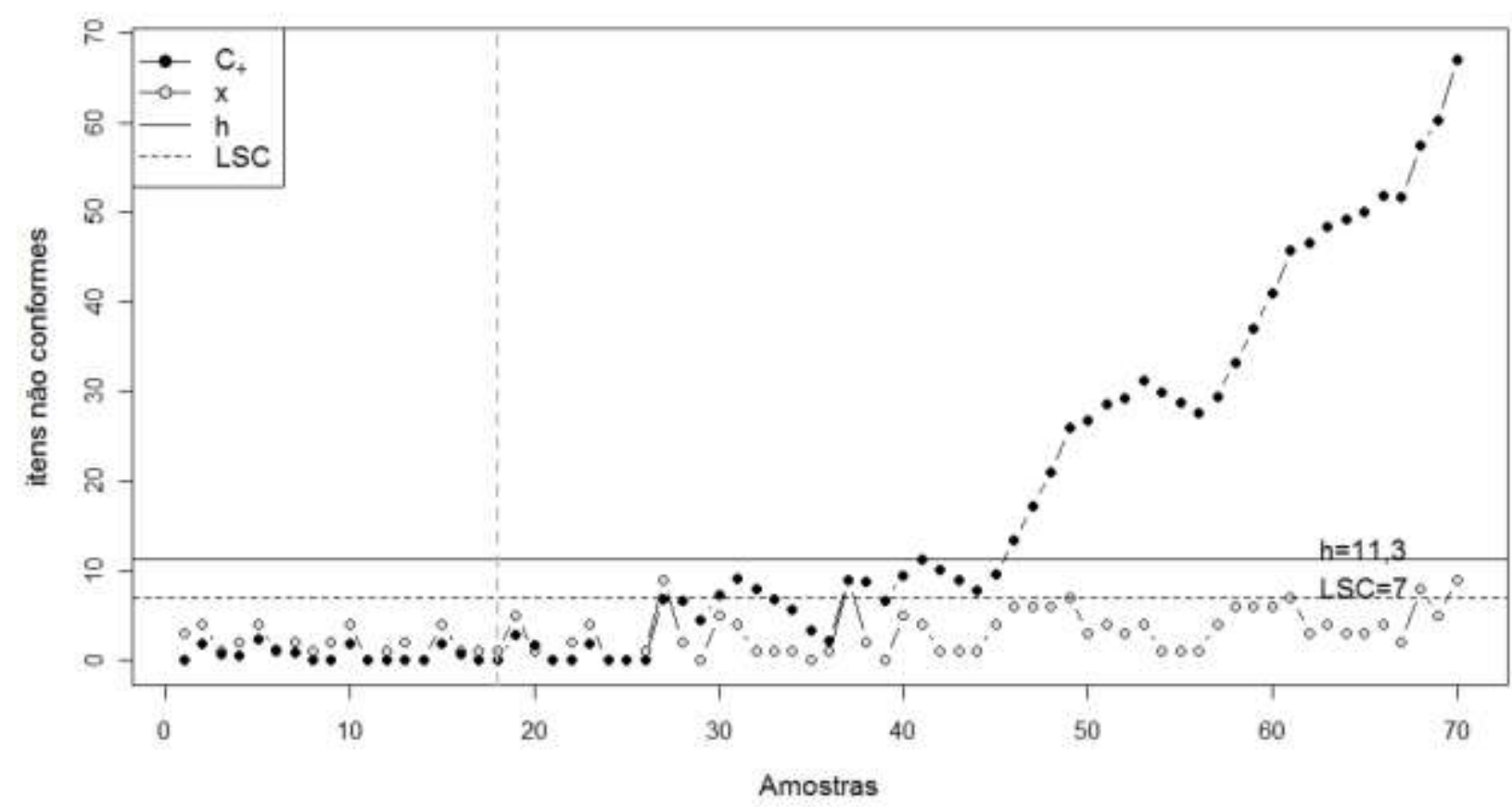

Fonte: Autoria própria (2011)

\subsection{Comparação com outros gráficos}

Para os mesmos dados e situação, $p_{1}=0,01$ e $A R L_{0}=443$, foi planejado e construído um gráfico CUSUM binomial (Figura 4). O valor de referência é o mesmo, $k=2,18$; e o limite superior é $h=10,6$. Por meio da Figura 4 pode-se verificar que este praticamente retorna as mesmas informações da parte CUSUM do gráfico combinado apresentado na Figura 3. 
Figura 4 - Gráfico CUSUM para os dados da rotulagem de garrafas

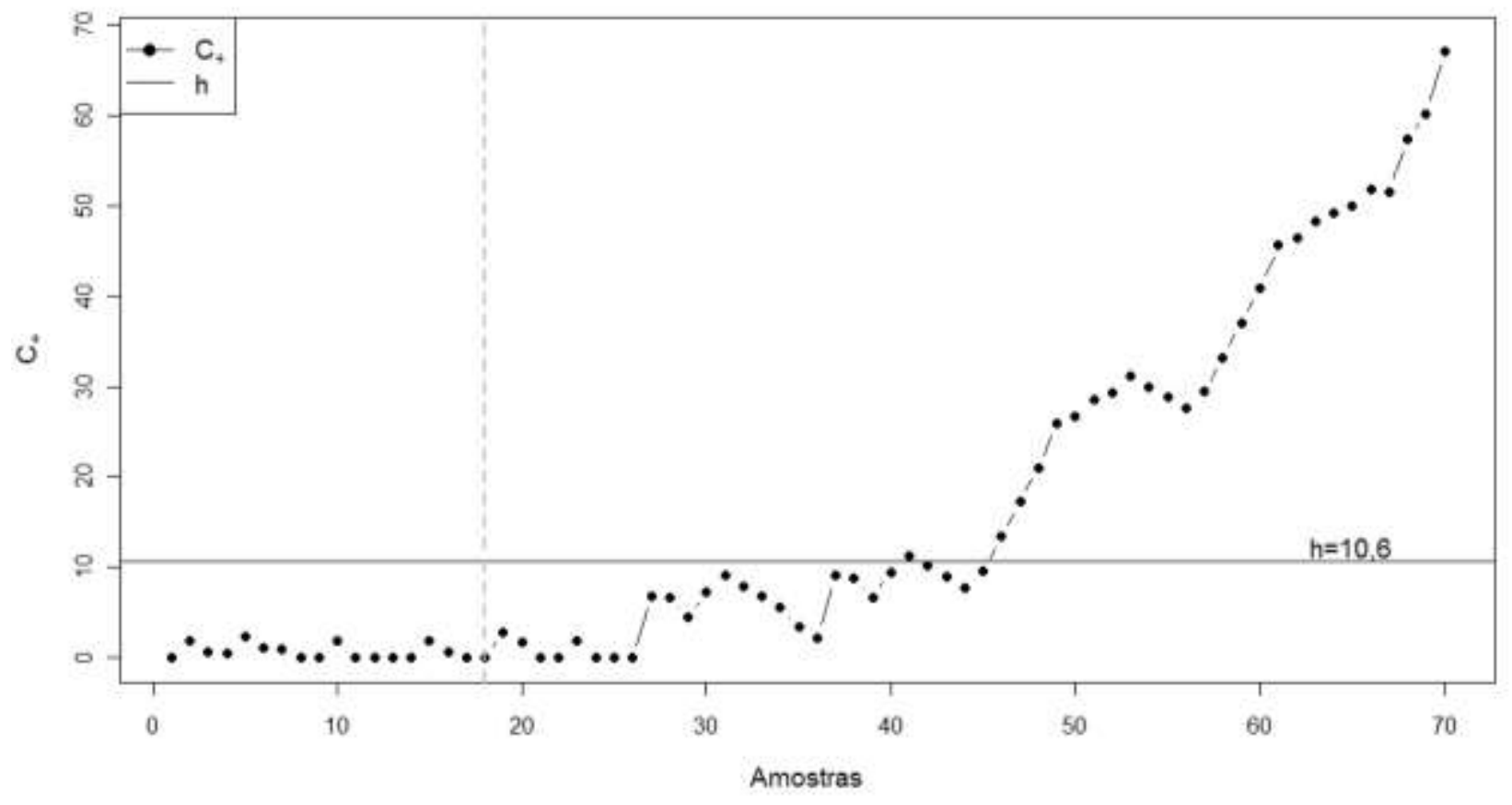

Fonte: Autoria própria (2011)

Em seguida (Figura 5) é analisado um gráfico do tipo Shewhart $n p$, com $L S C=6$. Pode ser visto que considerando apenas o limite superior, somente as amostras com mais itens defeituosos são sinalizadas e não há indicação do aumento da média do processo.

Figura 5 - Gráfico Shewhart para os dados da rotulagem de garrafas

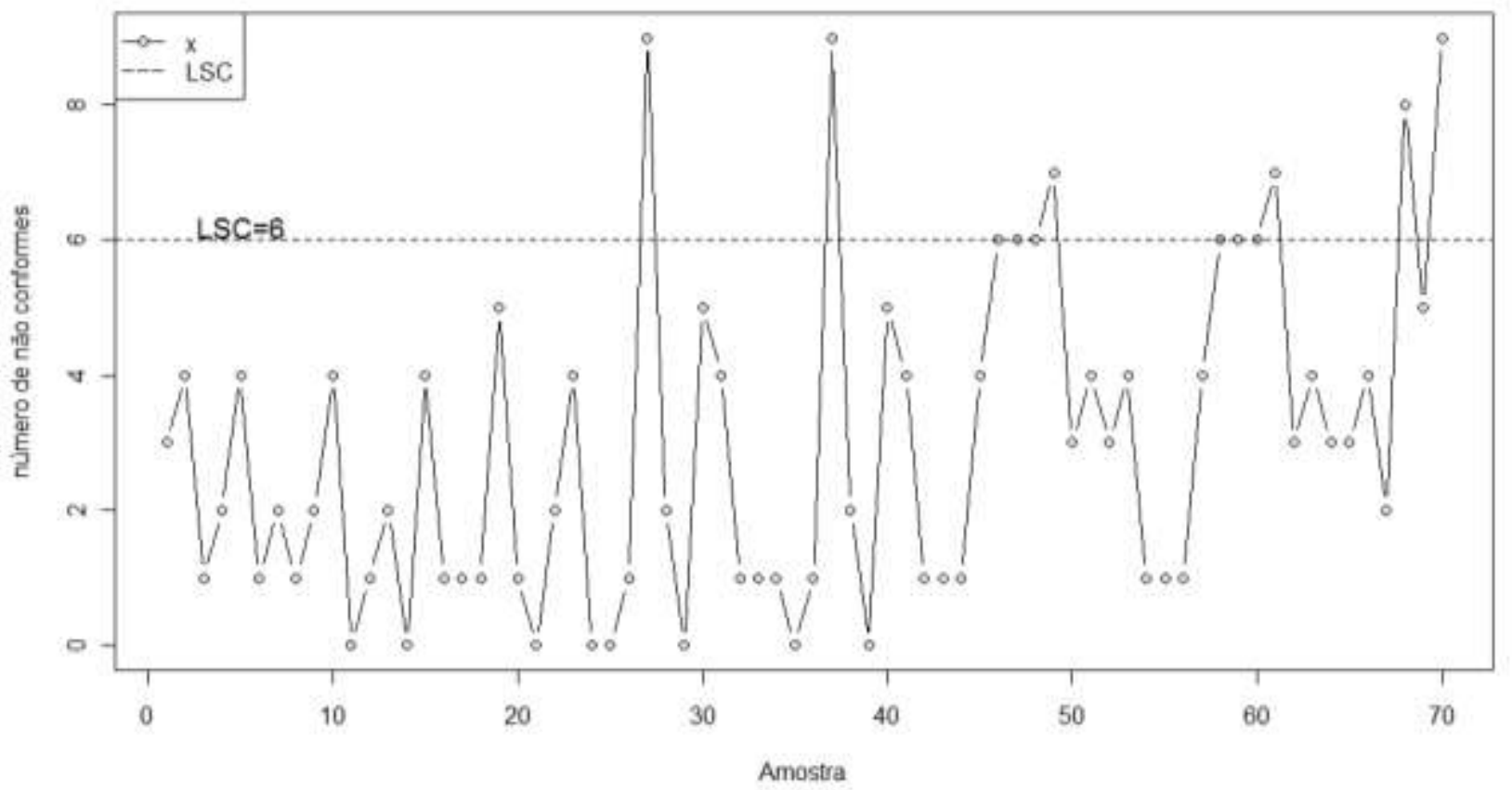

Fonte: Autoria própria (2011)

Na Figura 6 está um gráfico Shewhart com as regras sensibilizantes. Estas, sinalizadas no gráfico da Figura 6 como pontos em amarelo, dão suporte à idéia de que algo no processo está 
ocorrendo, mas não são diretamente explícitas quanto à tendência. Ressalta-se que a aplicação de regras sensibilizantes resulta também em menor valor de $A R L_{0}$, ou seja, a taxa de alarmes falsos aumenta.

Figura 6 - Gráfico Shewhart com regras sensibilizantes para os dados da rotulagem de garrafas

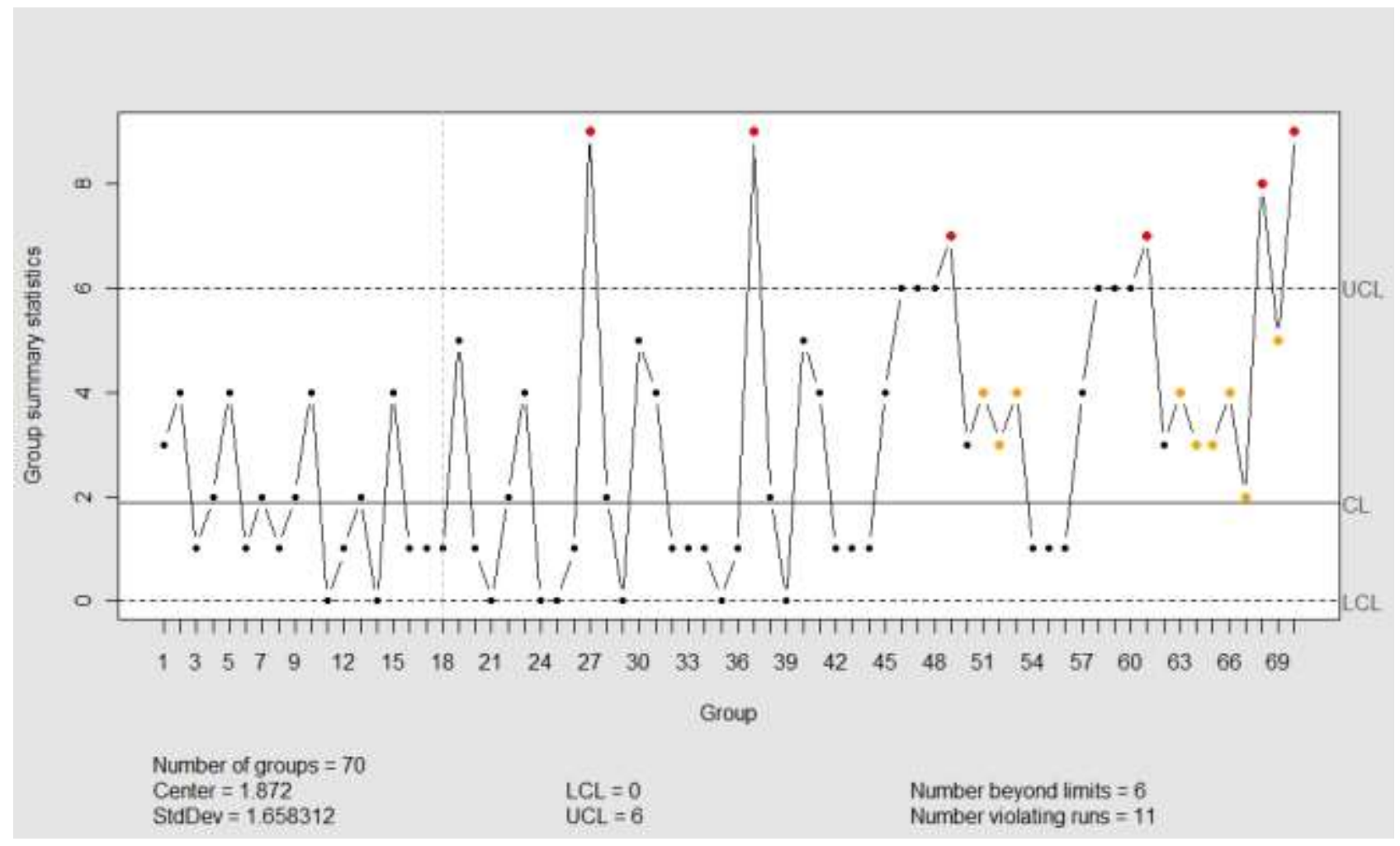

Fonte: Autoria própria (2011)

O gráfico combinado, para esta aplicação é uma opção a ser considerada. Teve o mesmo desempenho que o gráfico de Shewhart individual, na sinalização de alterações maiores e repentinas (amostras 27 e 41). E, comparado ao CUSUM, permitiu como este, detectar aumento na média $n p$ do processo.

\section{Conclusão e Considerações Finais}

A aplicação de um gráfico combinado Shewhart-CUSUM a este processo de rotulagem se mostrou uma alternativa interessante. O gráfico combinado captou as duas situações, alterações maiores (e repentinas) e menores, mas persistentes. O gráfico Shewhart, sem as regras suplementares, sinalizou apenas as mudanças de maior magnitude e repentinas. O gráfico CUSUM detectou a mudança no nível da média (np) do processo, mas deixou de sinalizar a amostra 27, onde $25 \%$ dos produtos eram defeituosos.

O gráfico combinado ainda foi comparado com um gráfico tipo Shewhart com regras sensibilizantes. No entanto, devem-se levar em conta as especificidades de cada processo e que o uso de regras suplementares tem as suas deficiências, já abordadas previamente neste trabalho. 
Na prática a aplicação de gráficos combinados pode ser de grande utilidade em processos onde a magnitude da mudança é desconhecida e há a necessidade de se detectar rapidamente tanto alterações pequenas a moderadas, quanto alterações maiores na proporção sob controle.

Este estudo ainda pode ser estendido, pois se limitou apenas a uma aplicação. A partir de procedimentos de simulação, por exemplo, seria possível avaliar situações abrangendo outros valores de $\mathrm{p}_{1}$ e também modificando a posição das mudanças repentinas.

\begin{abstract}
A combined Shewhart-CUSUM Control Chart corresponds to adding and adjusting the limits of a Shewhart type chart to a Cumulative Sum Control Chart (CUSUM). This combination aims to increase the sensitivity of the CUSUM chart for changes of greater magnitude. This article presents an application of a combined Shewhart-CUSUM binomial control chart in an industrial process of labeling bottles. Their performance was compared with the individual Shewhart and CUSUM charts. The combined chart is efficient because it signaled both sudden changes of greater magnitude, and a smaller increase, but gradual and persistent in the number of defective items, not flagged by the individual charts.
\end{abstract}

Key-words: Combined Shewhart-CUSUM Control Chart, binomial distribution, labeling bottles.

\title{
Referências
}

ABEL, V. On one-sided combined Shewhart-CUSUM control schemes for Poisson Counts. Computational Statistics Quarterly, v. 6, p. 31-39, 1990.

ALVES, C. C. Método da Equação integral com quadratura gaussiana para otimizar os parâmetros do gráfico de controle multivariado de somas acumuladas. 2009. 196 f. Tese (Doutorado em Engenharia de Produção). Programa de Pós-Graduação em Engenharia de Produção, Universidade Federal de Santa Catarina, Florianópolis.

ALVES, C. C.; SAMOHYL, R. W. A utilização dos gráficos de controle CUSUM para o monitoramento de processos industriais. In: ENCONTRO NACIONAL DE ENGENHARIA DE PRODUÇÃO, 24.,2004, Florianópolis. Anais... Florianópolis, UFSC, 2004.

BLACKSELL, S. et al. Use of combined Shewhart-CUSUM Control Charts in Internal Quality Control of Enzimelinked immunosorbent assays for the typing of foot and mouth disease virus antigen. Revue Scientifique et Technique (International Office of Epizootics), v.13, n.3, p. 687-699, 1994.

BLACKSELL, S. et al. Implementation of internal laboratory quality control procedures for the monitoring of ELISA performance at a regional veterinary laboratory. Veterinary Microbiology, v.51, p. 1-9, 1996.

COELHO, L. C. Utilização de modelos de suavização exponencial para previsão de demanda com gráficos de controle combinados Shewhart-CUSUM. 2008. 122f. Dissertação (Mestrado em Engenharia de Produção), Programa de Pós-Graduação em Engenharia de Produção, Universidade Federal de Santa Catarina, Florianópolis.

CHOU, C. J.; O'BRIEN, R. F.; BARNETT. D. B. Application of intrawell testing of RCRA groundwater monitoring data when no upgradient well exists. Environmental Monitoring and Assessment, n. 71, p. 91-106, 2001.

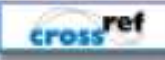

CONRATHS, F. J.; SCHARES, G. Validation of Molecular-diagnostic techniques in the parasitological laboratory. Veterinary Parasitology, v. 136, n. 2, p. 91-98, 2006. 
COSTA, A. F. B.; EPPRECHT, E. K.; CARPINETTI, L. C. R. Controle Estatístico de Qualidade. São Paulo: Atlas, 2004. 334 p.

CUI, H.; LUND, R. A new look at time series of counts. Biometrika. v. 96, n. 4, p. 781-792, 2009.

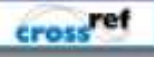

DU, J.; LI, Y. The integer-valued autoregressive (INAR(p)) model. Journal of Time Series Analysis, v.12, p. 129-42, 1991.

cross ref

GAN, F. F. An optimal design of CUSUM control charts for binomial counts. Journal of Applied Statistics, v. 20 , n. 4, p. 445-460, 1993.

cross ref

GAUTHIER, G.; LATOUR, A. Convergence forte des estimateurs des parameters d'un processus GENAR (p). Annales des sciences mathematiques du Quebec. v. 18, p. 37-59, 1994.

GIBBONS, R. D. Use of Combined Shewhart-CUSUM Control Charts for Ground Water Monitoring Applications. Ground Water, v. 5, n. 37, p. 682-692, set/out, 1999.

cross ref

HAWKINS, D.; OLWELL, D. Cumulative Sum Charts and Charting for Quality Improvement. New York: Springer Verlag Inc., 1998. 247 p.

cross ref

HENNING, E.; SAMOHYL , R. W.; ALVES, C. C. Aproximação do limite superior de um gráfico CUSUM binomial. In: ENCONTRO NACIONAL DE ENGENHARIA DE PRODUÇÃO, 30., 2010, São Carlos, Anais... São Carlos, 2010.

HENNING, E. Aperfeiçoamento e desenvolvimento dos gráficos combinados Shewhart-Cusum binomiais. 2010. 251f. Tese (Doutorado em Engenharia de Produção). Programa de Pós-Graduação em Engenharia de Produção, Universidade Federal de Santa Catarina, Florianópolis.

JONES, G.; GOVINDARAJU, K. A Graphical Method for Checking Attribute Control Charts Assumptions. Quality Engineering, v. 13, n. 1, p.19-26, 2000.

cross ref

LATOUR, A. Existence and Stochastic Structure of a Non-negative Integer-Valued Autoregressive Process. Journal of Time Series Analysis, v. 19, n. 4, p. 439-455, 1998.

cross ref

LUCAS, J. M. Combined Shewhart-CUSUM Quality Control Schemes. Journal of Quality Technology, v. 14, n. 2, p. $51-59,1982$.

MARI, L. The role of determination and assignment in measurement. Measurement, v. 21, n. 3, p. 79-90, 1997. cross ref

MEYER, D.; ZEILEIS, A.; HORNIK, K. ved: Visualizing Categorical Data. R package version 1.2-7, 2009. Disponível em: <http://www.r-project.org>. Acesso em: 06 jul. 2011.

MONTGOMERY, D. C. Introdução ao Controle Estatístico de Qualidade. 4. ed. Rio de Janeiro: LTC, 2004.

MORAIS, M. C. Ordenação estocástica na análise de desempenho de esquemas de controle de qualidade. 2002. 194f. Tese (Doutorado em Matemática) - Instituto Superior Técnico, Universidade Técnica de Lisboa, 2002. Disponível em:<http://www.math.ist.utl.pt/ mjmorais/thesis.pdf.>. Acesso em: 19 ago. 2008.

MORAIS, M. C.; PACHECO, A. Combined CUSUM-Shewhart Schemes for Binomial Data. Economic Quality Control, v. 21, n.1, p. 43-57, 2006. 
R DEVELOPMENT CORE TEAM. R: A language and environment for statistical computing. R Foundation for Statistical Computing, Vienna, Austria, 2011. Disponível em: <http://www.R-project.org>. Acesso em: 20 jan. 2011.

REYNOLDS, M. R.; STOUMBOS, Z. G. A CUSUM Chart for Monitoring a Proportion When Inspecting Continuously. Journal of Quality Technology, v. 31, n.1, p, 87-108, 1999.

REYNOLDS, M. R.; STOUMBOS, Z. G. Monitoring a Proportion using CUSUM and SPRT charts In : LENZ, H.J. WILRICH, P. Frontiers in Statistical Quality Control. v. 6. Springer Physika-Verlag, Heidelberg, p. 155-175, 2001.

ROCHA, R. Implementação de sistema gerencial, com avanços em controle estatístico, em laboratório de nutrição animal. 2004. 154f. Tese (Doutorado em Engenharia de Produção), Programa de Pós-Graduação em Engenharia de Produção, Universidade Federal de Santa Catarina, Florianópolis.

RIZZON, L. A.; MENEGUZZO, J. Sistema de Produção de Vinho Tinto. Embrapa Uva e Vinho. Sistemas de Produção, v.12, 2006. Disponível em: <

http://sistemasdeprodução.cnptia.embrapa.br/FontesHTML/Vinho/SistemaProducoaVinhoTinto/engarrafamento.htm.> Acesso em: 05 jul. 2010.

ROTONDARO, R. (Coordenador). Seis Sigma. Estratégia Gerencial para Melhoria de Processos, Produtos e Serviços. São Paulo: Atlas, 2002.

SAMOHYL, R. W. Controle Estatístico de Qualidade. São Paulo: Elsevier, 2009. 275 p.

SHARMA, P. R. A study of properties and applications of control charts for high yield processes. 2003. Tese (Doctor Of Philosophy) -Department of Industrial e Systems Engineering, The National University Of Singapore.

SILVA, I. M. M. Contributions to the analysis of discrete-valued time series. 2005. $212 \mathrm{f}$. Tese (Doutorado em Matemática) - Departamento de Matemática Aplicada, Faculdade de Ciências da Universidade do Porto. Disponível em: <http://paginas.fe.up.pt/ ims/phd_thesis_Isabel_Silva.pdf.>. Acesso em: 22 set. 2011.

SILVA, I.; SILVA, M. E. Seleção de ordem em modelos INAR. Actas do X Congresso Anual da Sociedade Portuguesa de Estatística. Edições SPE: Lisboa, p. 1-11, 2002.

SOLBERG, H. Monitoring long-term analytical quality by computerized combined Shewart-cusum method. Scandinavian Journal of Clinical and Laboratory Investigation. v. 172, p. 43-49, 1984.

SOUZA, G. P.; SAMOHYL, R. W. Monitoring Forecast Errors with Combined CUSUM and Shewhart Control Charts. Proceedings of the $26^{\text {th }}$. International Symposium of Forecasting, 2008.

TSUNG, F.; WANG, K. Adaptative Charting Techniques: Literature Rewiew and Extensions. In: LENZ, H.J. WILRICH, P. SCMID, W. Frontiers in Statistical Quality Control, v. 9. Springer Physika-Verlag, p. 19-36, 2010.

USEPA. Statistical Analysis of Ground-Water Monitoring Data at RCRA Facilities: Interim Final Guidance. Washington: Office of Solid Waste, 1989. Disponível em: 〈http: Iwww.epa.gov/otaq/transp/statanal.pdf.>. Acesso em: 10 set. 2008.

VINING, G. Technical Advice: Phase I and phase II control charts. Quality Engineering, v. 21, n. 4, p. 478-479, 2009. cross ref

VOSS, C.; TSIKRIKTSIS, N.; FROHLICH, M. Case Research in Operations Management. International Journal of Operations \& Production Management, v. 22 n. 2, p. 195-219, 2002.

eross ref

WESTGARD, James et al. Combined Shewhart-CUSUM Control Chart for Improved Quality Control in Clinical Chemistry. Clinical Chemistry, v. 23, n. 10, p. 1881-1887,1977.

WETHERILL, G. B.; BROWN, D. W. Statistical Process Control: Theory and Practice. Chapman and Hall, New York, 1991. 400 p.

WOODALL, W.; H. The Design of CUSUM Quality Control Charts. Journal of Quality Technology, v. 18, n. 2, p. 99-102, 1986. 
WU, Zhang et al. Optimization designs of the combined Shewhart-CUSUM control charts. Computational Statistics and Data Analysis, v. 53, n. 2, p. 496-506, 2008.

YASHCHIN, E. On the Analysis and Design of CUSUM-Shewhart Control Schemes. IBM Journal of Research and Development. v. 29, n.4, p. 377-391, 1985.

\section{cross ref}

YIN, R. K. Estudo de Caso: Planejamento e Métodos. 4.ed. São Paulo: Bookman, 2010.

ZHOU, Wanfang et al. Application of water quality control charts to spring monitoring in karst terranes.

Environmental Geology, v. 53, n. 6, p. 1311-1321, 2008.

\section{Anexo}

Tabela 1 - Conjunto de dados do processo de rotulagem de garrafas

\begin{tabular}{cccccccccc}
\hline NA & NC & NA & NC & NA & NC & NA & NC & NA & NC \\
\hline 1 & 3 & 15 & 4 & 29 & 0 & 43 & 1 & 57 & 4 \\
2 & 4 & 16 & 1 & 30 & 5 & 44 & 1 & 58 & 6 \\
3 & 1 & 17 & 1 & 31 & 4 & 45 & 4 & 59 & 6 \\
4 & 2 & 18 & 1 & 32 & 1 & 46 & 6 & 60 & 6 \\
5 & 4 & 19 & 5 & 33 & 1 & 47 & 6 & 61 & 7 \\
6 & 1 & 20 & 1 & 34 & 1 & 48 & 6 & 62 & 3 \\
7 & 2 & 21 & 0 & 35 & 0 & 49 & 7 & 63 & 4 \\
8 & 1 & 22 & 2 & 36 & 1 & 50 & 3 & 64 & 3 \\
9 & 2 & 23 & 4 & 37 & 9 & 51 & 4 & 65 & 3 \\
10 & 4 & 24 & 0 & 38 & 2 & 52 & 3 & 66 & 4 \\
11 & 0 & 25 & 0 & 39 & 0 & 53 & 4 & 67 & 2 \\
12 & 1 & 26 & 1 & 40 & 5 & 54 & 1 & 68 & 8 \\
13 & 2 & 27 & 9 & 41 & 4 & 55 & 1 & 69 & 5 \\
14 & 0 & 28 & 2 & 42 & 1 & 56 & 1 & 70 & 9 \\
\hline Nota: & & & & & & & & & \\
NA = número da amostra. & & & & & & & & \\
NC = número de garrafas onde o processo de rotulagem foi considerado não conforme.
\end{tabular}

\section{Dados dos autores:}

Nome completo: Elisa Henning

Filiação institucional: Universidade do Estado de Santa Catarina - UDESC

Departamento: Matemática - DMAT

Função ou cargo ocupado: Professor Adjunto

Endereço completo para correspondência: Rua Paulo Malschitzki, s/n, Campus Universitário Prof. Avelino Marcante, Bairro Zona Industrial Norte, Joinville, SC, Brasil. CEP 89219-710

Telefones para contato: (47) 40097978 
e-mail:dma2eh@joinville.udesc.br

Nome completo: Robert Wayne Samohyl

Filiação institucional: Universidade Federal de Santa Catarina - UFSC

Departamento: Engenharia de Produção e Sistemas - DEPS

Função ou cargo ocupado: Professor Titular

Endereço completo para correspondência: Caixa Postal 476, Trindade, Florianópolis, SC, Brasil.

CEP 88010-970

Telefones para contato: (48) 37217027

e-mail: samohyl@yahoo.com

Nome completo: Olga Maria Formigoni Carvalho Walter

Filiação institucional: Universidade Federal de Santa Catarina - UFSC

Departamento: Engenharia de Produção e Sistemas - DEPS

Função ou cargo ocupado: Mestranda do Programa de Pós-Graduação em Engenharia de Produção (PPGEP/UFSC)

Endereço completo para correspondência: Caixa Postal 476, Trindade, Florianópolis, SC, Brasil. CEP 88010-970

Telefones para contato: (47) 84287340 - (48) 37217055

e-mail: olgaformigoni@gmail.com

Nome completo: Rodrigo Leite Lindoso

Filiação institucional: Universidade Do Estado de Santa Catarina - UDESC

Departamento: Engenharia de Produção e Sistemas - DEPS

Função ou cargo ocupado: Acadêmico/Bolsista

Endereço: Rua Paulo Malschitzki, s/n, Campus Universitário Prof. Avelino Marcante, Bairro Zona

Industrial Norte, Joinville, SC, Brasil. CEP 89219-710

Telefones para contato: (47) 40097702

e-mail: rodrigoleite91@gmail.com

Enviado em: 23/09/2011

Aprovado em: 04/01/2013 